\title{
Space agency pushing for a bigger budget
}

\section{Washington}

THE financial cloud that has recently darkened space science in US universities is showing the first hint of a silver lining. The National Aeronautics and Space Administration (NASA) has told university researchers that its 1984 budget submission to the White House includes a strong plea for extra funds to help beleaguered university centres. If approved, NASA's rescue operation will include a special fund to modernize instruments, a new graduate fellowship programme for space science and a big increase in the amount of money available for analysing data from space missions.

In the view of most university space scientists, NASA's action is long overdue: a newly completed report drawn up by a joint NASA/university committee paints a startling picture of university departments unable to buy up-to-date equipment, short of graduate students and frustrated by the dearth of flight opportunities now that NASA's space science programme is focused on long-lived observatories and planetary orbiters. The committee, cochaired by NASA's chief scientist, Dr Frank McDonald, calls for immediate action to avert a crisis in university morale.

According to the group's report, NASA's relationship with the universities has undergone a profound change since the golden age of the $1960 \mathrm{~s}$. Then, the agency's space science programme involved four or five missions a year. NASA gave multidisciplinary research grants to more than 40 universities and supported construction of 37 space science buildings or additions. It also paid for a national programme of fellowships for graduate students; by the end of the decade, more than 5,300 students had received three-year awards.

Today, the launch rate of scientific satellites has dropped to one or two a year and the graduate fellowship programme and the university grants have stopped. The budget of NASA's Office of Space Science and Applications (OSSA), measured in constant dollars, has decreased from a peak of $\$ 1,630$ million in 1964 to $\$ 950$ million in 1984 . Because opportunities to fly experiments have at the same time decreased, 'drastic reductions' have been forced in many university space research groups.

Urging "extraordinary steps" to deal with the problem of ageing equipment, the report recommends that \$11 million a year be earmarked for five years from 1985 . The money would be spent on commercial laboratory equipment such as oscilloscopes and spectral analysers, small and medium size computers and major facilities such as micro-ion probes, gas analysers and large fast computers in the Cray class.

The report also recommends the immediate reintroduction of a NASA graduate research fellowship programme, beginning in 1985 with 50 fellowships and expanding to a level of 200 a year, to encourage bright undergraduates to pursue graduate work in sciences. Stipends would cover the full cost of tuition and include a living allowance of at least $\$ 13,000$ a year.

Finally, NASA wants a larger budget for data analysis and ground control. The report points out that the fall in the number of new missions has been partly offset by the increased longevity of spacecraft; at present, 14 active satellites are returning valuable new space science data, but funds to process the information have been scarce.

In 1983, the data analysis and mission operation part of the OSSA budget amounted to $\$ 155$ million, about 15 per cent of the total. The report proposes an increase of about $\$ 20$ million a year, with most of the extra money going to university groups. Some $\$ 10$ million would be allocated to Solar System exploration data, $\$ 4$ million to astrophysics and \$6 million to environmental observations.

If accepted by the Office of Management and Budget - and later by Congress -
NASA's package of spending increases will be a magnificent tonic for university space scientists. But the package will not deal with the problem that most worries university researchers, their diminishing access to space flights. In a stronglyworded letter to the committee, members of the University of Washington's McDonnell Center for the Space Sciences that unless new missions were conceived and flown, whole disciplines - such as $\mathrm{X}$-ray astronomy - could die.

Access to the shuttle has been the biggest disappointment. The Washington group claims that, when it wants to, NASA can help to mount experiments with remarkable speed. When the agency discovered last year that plastics were becoming dangerously eroded in space, it commissioned an emergency experiment, and the group was able to analyse the results only four months after being notified of the problem. But the group has now been told that it will be two years before it can fly another experiment.

In another letter to the committee, Dr Peter Banks, director of the Space Telecommunications and Radioscience Laboratory at Stanford University, predicts that the situation would not improve even when the Spacelab series is under way. The long delays and the emphasis on large-scale management and engineering projects put Spacelab experiments beyond the reach of most university groups. In the long run, he warned, NASA would dominate the development of space science facilities, with universities playing an ever diminishing part.

Peter David

\section{Biotechnology}

\section{Pessimism about patent rights}

\section{Washington}

BOTH patents and trade secrets may be "anachronisms", of little value in the fastpaced biotechnology industry, J. Leslie Glick, president of Genex, told a meeting of patent lawyers last week. Glick and several other speakers at the meeting, sponsored by the American Type Culture Collection (ATTC), said that traditional protection of intellectual property suffers from a number of failings when applied to biotechnology.

Inevitably, Glick said, there will be several solutions of a given problem in high-technology fields, thus providing competition against which patents or registration as trade secrets cannot protect. The typical three-year lag between the filing and issuance of a patent also limits the usefulness of patent protection.

The peculiar requirements that go with the award of biotechnology patents further tend to limit the scope of protection accorded. The legal requirement that a patent should fully disclose an invention, so that a person "skilled in the art" can reproduce it, must be met in the case of most biotechnology patents by depositing a sample of a novel organism in a public repository such as ATCC. These deposits, not required for patents in other areas, can "markedly reduce the research and development expenses" of a competitor seeking to improve on the invention or to evade the scope of the patented invention, according to Dale Hoscheit, a Washington patent lawyer.

Although hybridomas have been licensed as trade secrets they too present unusual problems. Limitations on free publication or on the exchange of materials among researchers is the most obvious drawback. Then state courts often require proof of even physical security before finding in favour of the owner of a trade secret who accuses a competitor of unlawfully taking his property - a condition not easily met when university laboratories are involved. And "reverse engineering", the reconstruction of a process from a knowledge of its endproduct, although not an immediate danger with hybridomas, is always a risk with trade secrets. 